MÁRGENES DE LA PSIQUIATRÍA Y HUMANIDADES

\title{
REFLEXIONES NERVIOSAS
}

\section{Chocolate.}

Los Archives of Internal Medicine publicaron el pasado mes de abril un trabajo de las norteamericanas Rose, Koperski y Golomb ${ }^{1}$ que pretende analizar la relación entre el consumo de chocolate y los síntomas depresivos. Las autoras indican que aunque existe todo un saber popular al respecto (plasmado en los más de 5.690.000 resultados de una búsqueda en Internet combinando los términos ingleses "chocolate" y "mood"), poco se ha investigado con rigor para confirmar tal relación y determinar su sentido. Por ello realizaron un análisis transversal sobre una muestra de 1018 mayores de 18 años (694 adultos y 324 adultas) de San Diego, California, con un buen estado de salud general, centrándose en los 931 sujetos que no tomaban antidepresivos y que facilitaron datos sobre su consumo de chocolate. Una vez los tuvieron rodeados y sin escapatoria posible, les administraron sin piedad un instrumento para la valoración de la depresión (el Center for Epidemiologic Studies Depression Scale, para los amigos, CES-D). Por si fuera poco, a los 839 probandos que se dejaron les pasaron el Fred Hutchinson Food Frequency Questionnaire para cuantificar su consumo de chocolate. Observaron que los sujetos con puntuaciones sugestivas de depresión (igual o superior a 16 en el CES-D) consumían más chocolate que los que se situaban por debajo de esa puntuación y lo hacían con una significación fetén $(\mathrm{P}=.004)$ aun más llamativa en sujetos que puntuabn por encima de 22 en el CES-D. Laás, común a varones y mujeres, era independiente de otros factores o hábitos dietéticos, por lo que Rose y colegas concluyen que a mayor puntuación de depresión en el citado instrumento, tanto mayor consumo de chocolate. Eso sí: consideran que no se puede establecer si existe una relación causal, y menos aún en qué dirección (es decir, que no nos aclaran si el chocolate causa o alivia la depresión). Tres años antes, los australianos Parker y Crawford ${ }^{2}$ ya habían realizado otro estudio, basado en un cuestionario online, en el que participaron 3.000 personas que decían padecer una depresión clínica. Los autores encontraron en la mitad de los participantes craving por chocolate (más notorio en damas que en caballeros). Además, su consumo fue considerado beneficioso para la depresión, la ansiedad y la irritabilidad y se asociaba a la presencia de rasgos indicativos de neuroticismo. El delicioso chocolate, por lo tanto, es algo más que un manjar; es una puerta abierta a la investigación más o menos científica sobre la psique humana. Impresionante.

Fue Linneo fue quien denominó al cacao "Theobroma", o "alimento de los dioses", término que tal vez guarde relación con el origen divino del producto que la mitología tolteca atribuye al producto, pero que según algunos autores lenguaraces podría deberse al apasionamiento por el chocolate que el insigne naturalista sueco con personajes tan variopintos como Calderón de la Barca, Francisco de Quevedo, Napoleón Bonaparte, Rubén Darío, el Papa Pablo VI, Agatha Christie, Neil Armstrong o, mejorando lo presente, un servidor.

El cacao es una planta de origen americano muy importante en la cultura y el comercio precolombino. Fue el propio Colón el primer europeo en reseñar que los centroamericanos usaban sus semillas como moneda. Los aztecas y mayas tenían en gran estima al cacao y a su infusión chocolatl. Confirmando que muchas veces los vencedores adoptan las 
MÁRGENES DE LA PSIQUIATRÍA Y HUMANIDADES

costumbres y hábitos de los vencidos, Hernán Cortés ordenó plantar cacao e introdujo la exótica bebida en la Corte de Carlos V en 1528. El conquistador consideraba al chocolatl una bebida muy apropiada para infundir vigor y lucidez a sus soldados.

A pesar de su sabor amargo para el gusto europeo, el chocolate consiguió extenderse por el Viejo Continente, gracias a que las clases altas hicieron de él una bebida de moda (el esnobismo y la tontería, pues, no son fenómenos recientes). A sus efectos tonificantes se le añadió una presunta capacidad afrodisíaca que lo hizo muy popular. Si Bernal Díaz del Castillo ya había informado de que Montecuhzoma Ilhuicamina tomaba cacao antes de visitar a sus numerosas concubinas, en Europa Madame du Barry lo servía a sus amantes antes de hacer el amor y para $\mathrm{Ca}$ sanova era más vigorizante que el champán. Con el verosímil afán de hacer más apetitosa tan cumplidora bebida, se sucedieron experimentos que la combinaban con sustancias tan diversas como la leche, el vino, la cerveza o la pimienta. No es de extrañar en este contexto que la Iglesia se movilizara contra el producto, prohibiera a los clérigos su uso y amenazara con la excomunión a quienes lo consumieran en misa (lo que hermana al chocolate con su "paisano" el tabaco, un producto consumido también por los sacerdotes durante la misa hasta que la Iglesia tomó cartas en el asunto).

El éxito definitivo del chocolate llegaría con su salto al estado sólido, debido a la prensa hidráulica del holandés Conraad Johannes van Houten, que no sólo permitía la elaboración del chocolate en polvo, sino que además aportó un método para separar la manteca de cacao, elemento indispensable para la tableta. Pasó así el chocolate de ser consumido preferentemente como bebida a convertirse en un delicado manjar sólido. Curiosamente, el café siguió un camino in- verso, ya que inicialmente se consumía en forma sólida. Y tal vez con cierta lógica, en paralelo a la solidificación del chocolate, a lo largo del siglo XIX el café se fue imponiendo como bebida de elección, relegando a la bebida de los dioses a una posición secundaria y más puramente hedónica en la dieta de los europeos.

En nuestros días las virtudes del chocolate no radican en su efecto vigorizante o afrodisiaco, sino en sofisticados y espesos razonamientos químicos. En el cacao encontramos un $30 \%$ de grasa, un $6 \%$ de proteínas, un $61 \%$ de carbohidratos, y un $3 \%$ de humedad y minerales de gran interés fisiológico, como magnesio, cobre, potasio, fósforo, calcio, hierro y zinc. También aporta vitamina A, vitamina B (niacina, pantoténico) y vitamina E. La manteca de cacao contiene un $35 \%$ de ácido oleico, un $35 \%$ de ácido esteárico y un $25 \%$ de ácido palmítico. El 5\% restante está integrado por diversos ácidos grasos, como el linoleico. El cacao es rico además en las tres metil-xantinas, a las que se atribuye su efecto tonificante. La principal metil-xantina del cacao es la teobromina $(1,2 \%)$ que estimula el músculo cardíaco y tiene un efecto vasodilatador. También se detectan feniletilamina, la inevitable serotonina y tiramina, así como otras sustancias menos inocentes.

Desde 1996 sabemos que el cacao contiene tres $\mathrm{N}$-aciletanolaminas (conocidas con la misericordiosa abreviatura de NEAs): la Noleoiletanolamina, la N-lineoiletanolamina y la andanamida, por orden decreciente de concentración ${ }^{3}$. Esta última, ligando endógeno de los receptores cannabinoides, debe su nombre al sánscrito ananda (bienestar). Su acción neurotransmisora permite augurar su posible utilidad para el desarrollo de nuevas terapias para la ansiedad, la depresión o el dolor. Las otras dos NEAs interrumpen el catabolismo de la andanamida, lo que potencia su acción provocando una leve acción 
cannabinomimética. Reconozcamos, por lo tanto, que quien en el argot de los consumidores tuvo la ocurrencia de designar al hachís como "chocolate" fue una persona inspirada y lúcida. Pero más recientemente se ha puesto en duda que el chocolate sea adictivo por esta vía. Por una parte, las NEAs se descomponen en el tubo digestivo antes de alcanzar la sangre o el cerebro ${ }^{4}$; además, el chocolate no contiene más andanamida que las avellanas, por lo que su efecto psicoadictivo ha de ser forzosamente menor que el de la marihuana (¿salvo tal vez en la combinación de chocolate con avellanas?). Hay además otros productos ricos en andanamida como la soja o la leche humana (se entiende que de mujer no consumidora de chocolate en cualquiera de sus acepciones) que no son adictivos. Por cierto, que este trabajo se debe a Vicenzo di Marzo y otros autores, procedentes del Instituto de Química Molecular de Nápoles y (es rigurosamente cierto) del Centro de Investigación de Nestlé. En otra aportación Smit y colaboradores ${ }^{5}$ concluyen que el efecto psicótropo del chocolate reside en las metilxantinas, más que en ingredientes calóricos. Lo que es una lástima, porque puestos a acusar al chocolate de ser una droga quedaría mucho más aparente y apropiado afirmar sin lugar a dudas que su acción psicológica se debe a una molécula emparentada con los cannabinoides.

Si nos remontamos más años atrás, mucho antes de que se hablase de las NEAs, el saber popular ya tenía claro que existía un craving por el chocolate e incluso una adicción al manjar divino, y se habían acuñado términos como "chocolohómano" o "chocohólico" para denominar a la persona que ¿sufría? tal adicción. En 1985, Favre-Bismuth y Grouzmann describieron la chocolatomanía, definiéndola como el consumo casi exclusivo de chocolate negro (con más del 50 $\%$ de cacao) en cantidades entre 100 y 500 gr/día, junto con una comórbida desmesura en actividades como los deportes, el ajedrez, el juego de cartas, las relaciones sexuales, la lectura o el cine, y una tendencia al ordenancismo $^{6}$. La diferenciaban de la bulimia por la ausencia de culpa, vómitos, uso de laxantes o alteraciones de la imagen corporal, y la caracterizaban como una práctica hedónica.

Clásicamente se ha afirmado que la querencia por el chocolate es más acusada entre las mujeres. Existen múltiples observaciones anecdóticas o naturalísticas, y otras de corte más científico, como el estudio que, con el apoyo -y no es broma- de la Federación Española de Asociaciones del Dulce y la Asociación Española de Fabricantes de Chocolate, validó el Cuestionario de Chocolates y Dulces ${ }^{7}$, versión castellana del Foods and Mood Inventary ${ }^{8}$. La investigación no sólo demostró que el instrumento está adornado de buenos valores de fiabilidad y consistencia interna, tanto para su sección de chocolate como para la de dulces, la experiencia, sino que arrojó el hallazgo colateral, pero no por ello menos significativo, de que las mujeres obtenían puntuaciones más altas que los varones.

Además, algunas observaciones señalan que la inclinación de las mujeres por el chocolate es cíclica y hormonodependiente. Casi la mitad de las norteamericanas experimentan craving por dulces y chocolate y la mitad de ellas lo hace especialmente en la proximidad de la menstruación. Un estudio de Michener y colaboradores estudió el consumo de un grupo de mujeres con ingesta perimenstrual de chocolate y/o dulces más o menos compulsiva y les administró de forma aleatorizada y en doble ciego, placebo, progesterona o alprazolam. Los autores no apreciaron cambios en el patrón de consumo o de craving con ninguno de los procedimientos, por lo que descartaron un desencadenante hormonal o afectivo ${ }^{9}$. Años después, Zellner 
MÁRGENES DE LA PSIQUIATRÍA Y HUMANIDADES

y asociados preguntaron a un colectivo de mujeres norteamericanas e hispanas cuándo se les exageraba el craving por chocolate, y si les sucedía así en torno a la menstruación. Las norteamericanas reconocían una mayor apetencia catamenial que no se daba entre las hispanas, lo que llevó a los autores a pensar que el craving femenino por el chocolate es más cultural que fisiológico ${ }^{10}$. Otros estudios, como el de Dallard y colaboradores ${ }^{11}$, encuentran que la adicción al chocolate no está tan sesgada hacia las mujeres, sino que se presenta en una población caracterizada por una vulnerabilidad hacia la depresión y la ansiedad, que emplea el producto para aliviar en parte su malestar, con dosis habitualmente estables, lo que diferencia la chocolatomanía de otras adicciones.

Más compleja, bastante más, es la hipótesis que Rezzi y colaboradores ${ }^{12}$ (vinculados también con el Instituto Nestlé) aventuraban en 2007 para justificar el gusto por el chocolate. Los autores defendían un nuevo enfoque "nutrimetabonómico" en el que correlacionaban fenotipos metabólicos generados mediante espectroscopia con preferencias dietéticas y conductuales; más concretamente con dos opuestas: "deseosa de chocolate " e "indiferente al chocolate". Caracterizaron los fenotipos metabólicos urinario y plasmático, incluido el perfil lipoproteico postprandial y el co-metabolismo de la flora intestinal. Los datos obtenidos sugerían que las diferentes preferencias dietéticas influyen en el estado metabólico basal y en la actividad del microbioma intestinal, quienes a su vez pueden determinar consecuencias a largo plazo para el huésped.

Y nos falta la vía de la neuroimagen para caracterizar y si es posible anatemizar el consumo de chocolate. En 2004, Wang y colaboradores publicaron en Neuroimage un estudio $^{13}$ con 12 personas sanas, no obesas ni bulímicas, ni adictos as sustancias, a las que se expuso a la contemplación, olor y sabor de sus alimentos favoritos, incluido el chocolate. Con las pertinentes imágenes en colorines generadas por sofisticados cacharricos se comprobó que la experiencia activaba en los probandos regiones cerebrales que se excitan cuando las personas adictas a la cocaína piensan en su consumo. En concreto, esta exposición a sus alimentos favoritos aumentó notablemente (en un $24 \%$ ) el metabolismo especialmente en la ínsula anterior, la circunvolución temporal superior y la corteza orbitofrontal (COF). La participación de esta última zona permitió a los autores proponer que la publicidad de los alimentos repercute en la epidemia de obesidad que afecta a Estados Unidos. Cierto es que tanto colorín y toda las alusiones a recónditas esquinas cerebrales y a autopistas dopaminérgicas impone bastante, pero uno no le queda más remedio que preguntarse cuándo se estudiará lo que sucede en la COF de filatélicos expuestos a sellos raros o deseables, en seguidores de un determinado equipo de fútbol ante los primeros toques de balón por parte del crack de turno, o en general cuando se expone a cualquier persona a una situación que le resulte apetecible o placentera más allá del ámbito de las drogas o las dietas.

Para que el razonamiento sobre el craving quede redondo falta la terapéutica farmacológica. En 1989 el American Journal of Psychiatry publicó una carta en la que el doctor Michell y asociados ${ }^{14}$ relataban el hallazgo serendípico de dos pacientes depresivos con craving por chocolate comórbido en los que la querencia había desaparecido a los pocos días de iniciar el tratamiento con bupropion. Los autores descartaban la adicción fuera secundaria a la depresión o que la querencia desapareciera al mejorar el cuadro afectivo, por lo que concluían que la respuesta tenía que estar en el antidepresivo. Como faltaban aún tres años para que se descubriera la anda- 
namida y quince para que se atribuyese toda la acción psicotrópica del producto a las metilxantinas, no tuvieron más alternativa que fijarse en que la estructura química del bupropion era clavadita a la de la feniletilamina del chocolate. De esta manera, el antidepresivo produciría en los pacientes una "saciación" ante la que no tendría lugar ni sentido el ansia por chocolate. Pasados unos meses, Rakatansky ofrecía una explicación alternativa ${ }^{15}$ : en su opinión Michell y colaboradores habían descubierto un efecto secundario grave del bupropion consistente en la abolición del placer de consumir chocolate. ¿Qué sería de quienes obtienen un beneficio hedonista del chocolate si se les privara a golpe de psicofármaco de su placentero consumo?, se preguntaba, al tiempo que imaginaba un escenario pavoroso en el que la gente, abandonado el chocolate, se entregaría a otras actividades, como el sexo (con el consiguiente riesgo de un baby-boom) o -peor aún- al ejercicio compulsivo. Aunque estas alternativas, a la larga, pudieran ser provechosas, en el plazo corto generarían una necesidad de asistencia psiquiátrica con un enorme despliegue de recursos humanos que pondría a la sanidad al borde del colapso económico. Por todo ello, sugería que la FDA prohibiera el bupropion o limitara su prescripción, y se preguntaba cuándo cesaría el afán de lo que llamaba farmacología creativa por modelar la conducta alimentaria de la ciudadanía, que a su modo de ver, tenía un incómodo regusto orwelliano.

Tanta insistencia sobre la presunta adicción al chocolate pone de manifiesto que los seres humanos somos gente de pocas ideas, que vuelve sobre ellas con tenacidad y una especie de atavismo del que somos incapaces de liberarnos. La Historia de la Medicina demuestra que los límites entre las ideas de enfermedad y vicio son muy borrosos. También permite ver que ciertas causas -reales o presuntas- de las enfermedades han servido para destacar, por encima del dolor o el sufrimiento del paciente, las conductas previas que según el momento histórico se han previas relacionado tan alegre como dogmáticamente con la aparición de la enfermedad. En la época moderna, podemos recordar las peregrinas teorías masturbatorias sobre la histeria -a las que llegó a apuntarse el mismísimo Freud- o la epilepsia. Más recientemente, la terapéutica antiinfecciosa ofreció al enfermo la posibilidad de curación e incluso de redención. Pero si bien la irrupción de la penicilina redimió a quienes padecían enfermedades venéreas hasta entonces fatales, la llegada de la infección VIH rescataría la vieja idea de la enfermedad como retribución al depravado, sin redención posible ${ }^{16}$. La toma continua de antirretrovirales y sus molestos secundarismos equivalen, por lo tanto, a una penitencia por el mal causado al propio cuerpo con una conducta licenciosa e inmoral. Más aun: la lipodistrofia generada por alguno de estos productos podría representar un modero estigma comparable a las llagas del leproso, el apestado y marginado clásico.

Pero en nuestra época lo médicamente pecaminoso se ha desplazado desde la esfera sexual hacia otros atentados contra la pureza corporal. Destacan entre ellos los hábitos considerados insanos desde el punto de vista metabólico o cardiovascular, auténticos vicios posmodernos que se contraponen a los virtuosos hábitos saludables en la alimentación y las costumbres. La cuestión llega hasta el punto de que el ser humano, siempre desmedido y proclive al fundamentalismo, ha generado formas fanáticas, por exceso, de estos hábitos saludables que, unidas al celo descriptivo y analítico de la Medicina, han permitido la identificación de dos nuevas especies morbosas: la ortorexia y la vigorexia.

La dualidad de los hábitos saludables (mesurada o desbocada, que remite a la di- 
MÁRGENES DE LA PSIQUIATRÍA Y HUMANIDADES

cotomía clásica de templanza y desenfreno) tiene su paralelo en la clásica y a veces olvidada dualidad de los fármacos, en los que se combina una acción terapéutica deseada con toda una amplia gama de efectos colaterales y secundarios inoportunos e indeseables. Los griegos acuñaron el término phármakon que resultaba muy conveniente para designar a drogas y medicamentos, ya que incorporaba significados contrapuestos como remedio o veneno. El término podría aplicarse también a los alimentos, que vienen siendo objeto desde hace años del interés de una literatura científica y divulgativa deseosa de explorar su capacidad "terapéutica" o preventiva. Así, al efecto ansiolítico y antidepresivo por potenciación serotoninérgica atribuido a los carbohidratos, hay que añadir que la glucosa oral a concentraciones bajas, tiene un efecto analgésico superior al de los anestésicos en neonatos sometidos a venopunción ${ }^{17}$. Puesto que los carbohidratos también estimulan el sistema colinérgico, el desayuno copioso se ha convertido en un principio alimentario saludable básico. Por su parte, los aminoácidos se han usado como antidepresivos, como muestra el uso en Psiquiatría del Triptófano y la S-Adenosil-Metionina. Y las grasas, en particular los ácidos omega-3, amén de sus virtudes sobre el aparato circulatorio, se propugnan desde hace tiempo como reguladores del humor y potenciadores de antidepresivos $^{18}$. También se ha asegurado que podrían tener una acción preventiva sobre el deterioro cognitivo, aunque su magnitud es incierta $^{19}, y$ por si fuera poco, este mismo año se ha asegurado que son capaces de prevenir la irrupción de trastornos psicóticos ${ }^{20}$. El potencial nocivo de carbohidratos y proteínas, según la dosis y las condiciones del paciente, es incluso más evidente en las grasas, lo que justifica que conceptualicemos como phármakon también a los alimentos. Entre ellos, claro, al chocolate.
A la larga tradición, ya expuesta, sobre los efectos psicotrópicos, reales o supuestos (euforizante, vigorizante, afrodisíaco) del chocolate, hay que añadir su posible efecto preventivo de algunas enfermedades, en especial las cardiovasculares. La idea surgió cuando se observó que el bajo riesgo de hipertensión de los indios kuna de las islas próximas a Panamá pasaba a equipararse al de la población general entre los kuna emigrados al continente. Para dar respuesta al enigma se propuso que los isleños consumen cacao puro, rico en procianidinas, mientras el chocolate industrial continental que comen los emigrados es mucho menos rico en estas sustancias $^{21}$, cuya capacidad antioxidante es incluso superior a la de otros reputados productos como el vino tinto, el té verde y el té negro. La riqueza del cacao en procianidinas augura prometedoras virtudes preventivas de patologías cardiovasculares y cancerosas ${ }^{22},{ }^{23}$ que invitan a incluirlo, en cantidades siempre moderadas (frente a la gula, templanza), en la dieta saludable ${ }^{24}$ que todo ciudadano consciente y responsable ha de seguir para alcanzar la pureza del cuerpo, ahora que no se lleva tanto la del alma. Por si fuera poco, podemos registrar más beneficios sobre el sistema cardiovascular gracias a un estudio holandés que observó que la ingesta habitual de cacao se asociaba con una menor tensión arterial y una reducción, en quince años, quince, de seguimiento, de la mortalidad no sólo cardiovascular, sino de cualquier origen $^{25}$. Y por si fuera poco, un metaanálisis de Taubert ${ }^{26}$ y asociados confirmó que posee un efecto hipotensor que no se objetiva en el té, que además no es ni de lejos tan rico como el chocolate.

La bibliografía científico - médica nos ofrece más ventajas del cacao. Como hacen notar Castillejo y colaboradores, es un remedio excelente (y de mejor sabor que otros productos competidores) para el estreñimien- 
MÁRGENES DE LA PSIQUIATRÍA Y HUMANIDADES

to infantil ${ }^{27}$, y por si fuera poco, que no lo es, la teobromina (es decir, la metil-xantina característica del cacao) es un antitusígeno mejor que la codeína, lo que le permite bloquear la tos producida experimentalmente por capsaicina, posiblemente al inhibir la despolarización del nervio vago desencadenada por esta sustancia ${ }^{28}$. Dentro de la miscelánea de efectos beneficiosos del chocolate debemos incluir las conclusiones de un peculiar estudio finlandés en el que se entrevistó a 305 madres de niños sanos para comprobar si el consumo de chocolate y las experiencias estresantes durante el embarazo guardaban relación con el temperamento del bebé a los seis meses de vida. A pesar de todo el artefacto que puede derivarse de que las valoradoras del carácter eran las propias madres, los autores observaron que quienes habían comido chocolate a diario durante la gestación percibían de forma más positiva el temperamento de sus hijos. En cambio, las valoraciones negativas se asociaban a un mayor estrés materno preparto, en especial en las mujeres que habían ingerido poco chocolate. No es de extrañar que los autores señalen que "además de producir sentimientos subjetivos de bienestar psicológico", el chocolate "puede tener efectos en múltiples niveles ambientales y psicológicos", como si su consumo hubiera protegido frente al estrés a las madres propiciándoles una mayor armonía con sus hijos $12^{29}$. Una explicación alternativa, que eludiría hipótesis psicofarmacológicas es que el consumo de chocolate sería más bien un "marcador" de una actitud general optimista frente a la vida. Pero sea cual fuere la razón de sus hallazgos, que levante el dedo quien se atreva a negar o condenar un antojo chocolatero en una embarazada.

Pero el chocolate, como phármakon que es, también efectos secundarios y colaterales, como su acción hiperinsulinemiante postprandrial $^{30}$, de significado incierto, tanto más cuanto un estudio posterior demostró que los flavonoides del cacao mitigan los efectos negativos de la diabetes sobre el endotelio $^{31}$, confiriendo de nuevo al cacao un efecto protector sobre el sistema cardiovascular. Y se imputan más cargos al chocolate. El proyecto "Mood and Food", auspiciado por la asociación británica Mind, afirma que existen alimentos que estabilizan el estado de ánimo (agua, verduras, fruta, pescado rico en ácidos grasos, frutos secos, cereales integrales, fibra, proteínas y alimentos ecológicos), en tanto que otros como el chocolate (junto al azúcar, la cafeína, el alcohol, los cereales industriales, los aditivos, la leche y las grasas saturadas), producen excitación y estrés ${ }^{32}$. También se dice que la cafeína del chocolate podría fomentar las pesadillas y los trastornos conductuales ligados al sueño REM ${ }^{33}$.

La relación entre el chocolate y la Medicina tiene una dimensión especial que no conviene pasar por alto. Regalar bombones es una de las maneras en que los pacientes, en especial los hospitalarios, expresan su agradecimiento y reconocimiento al personal sanitario. Un trabajo publicado un número navideño y ganso del BMJ y realizado en Gales $^{34}$, estudió si la exposición a los bombones y a su degustación a lo largo de años de experiencia profesional podría ser proporcional a la habilidad para identificarlos. Para evaluar su hipótesis, los investigadores presentaron una caja de bombones a diferentes profesionales a los que pidieron que denominaran cada clase de bombón y definieran su sabor. Las enfermeras (habituales degustadoras del producto) fueron especialmente brillantes en la tarea, al igual que los residentes de mayor cualificación, mientras que los residentes más bisoños y el personal médico más experimentado carecían de un profundo conocimiento bombonero. La impericia de los médicos más jóvenes puede explicarse por una u menor experiencia que hace que hayan 
MÁRGENES DE LA PSIQUIATRÍA Y HUMANIDADES

probado menos bombones que sus compañeros de mayor grado o que las enfermeras. En cambio, los especialistas, a pesar de su incapacidad para nombrar la clase de bombón fueron muy capaces de identificar su sabor, en un claro paralelismo con esa "intuición" u "ojo clínico" (en este caso, sería nariz clínica) del médico veterano, que le permite "oler" las enfermedades o los diagnósticos aunque no razone su impresión con los algoritmos al uso. Los autores sugieren que la pérdida de la capacidad de nombrar la marca o clase del bombón es un marcador indirecto del tiempo de presencia del profesional en la planta, por lo que el test de identificación de bombones, a pesar de su apariencia inocente, sería una manera sencilla, eficaz y perversa de medir el grado de escaqueo de los profesionales (que en Gales, al parecer, distingue sobre todo a los especialistas veteranos). Conviene estos hallazgos en nuestro medio, porque al hilo de la resistencia que suscita a veces fichar a la entrada y salida de los hospitales, a uno se le ocurre que los gestores bien podrían sustituir la maquinita en cuestión por un test de reconocimiento de bombones mucho más llevadero y agradable (friendly, que se dice) e no menos eficaz para pillar al profesional absentista y escaqueador. Es una idea, nada más.

Y remataremos la cuestión con el toque biotecnológico, que ahora se lleva mucho. Lynne MacKaskie y sus colegas de la Universidad de Birmingham consiguieron, hace unos años, obtener energía de los restos de dulces y chocolates ${ }^{35}$. Utilizaron para ello la siempre socorrida $E$. Coli, que gracias a su hidrogenasa metaboliza el azúcar en hidrógeno y diversos ácidos. En la experiencia que comentamos el hidrógeno resultante fue capaz de impulsar un pequeño ventilador. Para tranquilidad de los amantes del chocolate y los dulces, aclararemos que por el momento, el procedimiento está aún en mantillas, por lo que nadie debe aún temer que en aras de la obtención de energía se proponga destinar a la E. Coli el chocolate de consumo humano. $\mathrm{Y}$ es que excepción hecha de las bacterias, parece que los primates somos la especie más apta para comer chocolate. Animales con los que los humanos compartimos el nicho cultural, como los perros pueden morir envenenados por el chocolate que tanto les gusta. Tan severa toxicidad se debe a la teobromina, dañina para el músculo cardiaco de los cánidos, hasta el punto de que se ha ensayado el chocolate enriquecido en esta metil-xantina para eliminar plagas de coyotes ${ }^{36}$. Si recordamos que teobromina remite etimológicamente a alimento de los dioses, el dato de que otras especies mueren intoxicadas por el satisfará sin duda al característico narcisismo y antropocentrismo humano.

Juan Medrano

\section{BIBLIOGRAFÍA:}

(1) Rose N, Koperski S, Golomb BA. Mood Food. Chocolate and Depressive Symptoms in a Cross-sectional Analysis. Arch Int Med 2010; 170: 699-703 [Resumen en línea]

(2) Parker G, and Crawford J. Chocolate craving when depressed: a personality marker. Br J Psychiatry 2007; 191, 351-2 [Texto en línea]

(3) Di Tomaso E, Beltramo M, PiomeLLI D. Brain cannabinoids in chocolate. Nature. 1996; 382: 677-8 [Texto en línea].

(4) Di Marzo V, Sepe N, De Petrocellis L, Berger A, Crozier G, Fride E, MeCHOULAm R. Trick or treat from food endocannabinoids? Nature. 1998; 396:636-7 [Resumen en línea]. 
MÁRGENES DE LA PSIQUIATRÍA Y HUMANIDADES

(5) Smit HJ, Gaffan EA, Rogers PJ. Methylxanthines are the psycho-pharmacologically active constituents of chocolate. Psychopharmacology (Berl) 2004; 176: 412-9 [Resumen en línea].

(6) Favre-Bismuth C, Grouzmann E. Chocolatomanies. A propos de 22 cas. Entretiens de Bichat 1985; 108-11.

(7) Bulbena Vilarrasa A, GonzálezPinto Arrillaga A, Martín-Santos Laffon R, Guimón Ugartechea J, Dasquens Soler J. Adaptación castellana y estudio factorial del cuestionario de chocolate y dulces (CCHD). An Psiquiatría (Madrid) 1993; 9: 251-255.

(8) Schuman M, Gituin MJ, Fairbanks L. Sweets, chocolate and atypical depressive traits. J Nerv Ment Dis 1988; 175: 491-495 [Resumen en línea]

(9) Michener W, Rozin P, Freeman E, GaLE L. The role of low progesterone and tension as triggers of perimenstrual chocolate and sweets craving: some negative experimental evidence. Physiol Behav 1999; 67: 4 17-20 [Resumen en línea]

(10) Zellner DA, Garriga-Trillo A, Centeno S, Wadsworth E. Chocolate craving and the menstrual cycle. Appetite 2004; 42: 11921 [Resumen en línea].

(11) Dallard I, Cathebras P, Sauron C, Massoubre C. Le cacao, est-il un psychotrope? Étude psychopathologique dúne population s'identifiant comme chocolatomane. Encephale 2001; 27: 181-6 [Resumen en línea]

(12) Rezzi S, Ramadan Z, Martin FP, Fay LB, van BLAdEREN P, Lindon JC, et al. Human metabolic phenotypes link directly to specific dietary preferences in healthy individuals. J Proteome Res 2007; 6: 4469-77 [Resumen en línea]

(13) Wang GJ, Volkow ND, Telang F, ET AL. Exposure to appetitive food stimuli markedly activates the human brain. Neuroimage 2004; 21: 1790-7 [Resumen en línea]

(14) Mitchell GF, Mebane AH, BI-
LLINGS CK: Effects of bupropion on chocolate craving. Am J Psychiatry 1989; 148, 812

(15) RaKatansky H. Chocolate: pleasure or pain? Am J Psychiatry 1989; 146: 1089.

(16) Bud R. Antibiotics. From germophobia to the carefree life and back again: the lifecycle of the antibiotic brand. En: Tone E, Siegel Watkins E. Medicating modern America. Prescription drugs in history. New York: New York University Press; 2007. p 17-41

(17) Gradin M, Ericsson M, HolmQvist G, Holstein A, Schollin J. Pain reduction at venipuncture in newborns: oral glucose compared with local anesthetic cream. Pediatrics 2002; 110: 1053-7 [Resumen en línea]

(18) Peet M, Horrobin DF. A dose-ranging study of the effects of ethyl-eicosapentaenoate in patients with ongoing depression despite apparently adequate treatment with standard drugs. Arch Gen Psychiatry. 2002; 59: 913-9 [Resumen en línea]

(19) Daviglus ML, Bell CC, BerrettiNi W, Bowen PE, Connolly ES, Cox NJ, et al. NIH State-of-the-Science Conference Statement: Preventing Alzheimer's Disease and Cognitive Decline. NIH Consens State Sci Statements 2010; 27(4) [Texto en línea]

(20) AMminger GP, SchäFer MR, PAPAgeorgiou K, Klier CM, Cotton SM, Harrigan SM, ET AL. Long-chain omega-3 fatty acids for indicated prevention of psychotic disorders: a randomized, placebo-controlled trial. Arch Gen Psychiatry 2010; 67: 146-54 [Resumen en línea].

(21) Hollenberg N. Vascular action of cocoa flavanols in humans: the roots of the story. J Cardiovasc Pharmacol. 2006; 47 Suppl 2: S99102 [Texto en línea].

(22) Weisburger JH. Chemopreventive effects of cocoa polyphenols on chronic diseases. Exp Biol Med (Maywood). 2001; 226: 891-7 [Texto en línea].

(23) WAN Y, Vinson JA, ETHERTON TD, Proch J, Lazarus SA, Kris-Etherton PM. 


\section{MÁRGENES DE LA PSIQUIATRÍA Y HUMANIDADES}

Effects of cocoa powder and dark chocolate on LDL oxidative susceptibility and prostaglandin concentrations in humans. Am J Clin Nutr 2001; 74: 596-602 [Texto en línea].

(24) Steinberg FM, Bearden MM, KeEn CL. Cocoa and chocolate flavonoids: implications for cardiovascular health. J Am Diet Assoc 2003; 103: 215-23 [Resumen en línea].

(25) Buisse B, Feskens EJ, Kok FJ, Kromhout D. Cocoa intake, blood pressure, and cardiovascular mortality: the Zutphen Elderly Study. Arch Intern Med 2006; 166: 411-7 [Texto en línea].

(26) Taubert D, Roesen R, Schomig E. Effect of cocoa and tea intake on blood pressure: a meta-analysis. Arch Intern Med 2007; 167: 626-34 [Texto en línea].

(27) Castillejo G, Bulló M, Anguera A, Escribano J, Salas-Salvadó J. A controlled, randomized, double-blind trial to evaluate the effect of a supplement of cocoa husk that is rich in dietary fiber on colonic transit in constipated pediatric patients. Pediatrics 2006; 118: e641-8 [Texto en línea]

(28) Usmani OS, Belvisi MG, Patel HJ, Crispino N, Birrell MA, Korbonits M, KorboNITS D, BARNES PJ. Theobromine inhibits sensory nerve activation and cough. FASEB J. 2005; 19: 231-3 [Texto en línea].

(29) Räikkönen K, Pesonen AK, JärVEnPäÄ A-L, Strandberg TE. Sweet babies: chocolate consumption during pregnancy and infant temperament at six months. Early Hum Dev 2004; 76: 139-45 [Resumen en línea].
(30) Brand-Miller J, Holt SHA, de Jong V, Petocz P. Cocoa powder increases postprandial insulinemia in lean young adults. $\mathrm{J}$ Nutr. 2003; 133: 3149-52 [Texto en línea].

(31) Balzer J, Rassaf T, Heiss C, Kleinbongard P, Lauer T, Merx M et al. Sustained Benefits in Vascular Function Through Flavanol-Containing Cocoa in Medicated Diabetic Patients. A Double-Masked, Randomized, Controlled Trial. J Am Coll Cardiol 2008; 51: 2141-9 [Texto en línea].

(32) http://www.mind.org.uk/foodandmood/ http://www.mind.org.uk/foodandmood/, accedido el 20 de junio de 2010

(33) Vorona RD, Ware JC. Exacerbation of REM sleep behavior disorder by chocolate ingestion: a case report. Sleep Med. 2002; 3 : 365-7 [Resumen en línea].

(34) Cooke F, Morse R. Do you know your chocolates? Recognition survey among medical staff of various grades. BMJ 1997; 315: 1655-1656. [Texto en línea]

(35) Macaskie LE, BaXter-Plant VS, Creamer nJ, Humphries aC, Mikheenko IP, Mikheenko PM, ET AL. Applications of bacterial hydrogenases in waste decontamination, manufacture of novel bionanocatalysts and in sustainable energy. http://www.biochemsoctrans.org/ bst/033/0076/bst0330076.htm" Biochem Soc Trans 2005; 33: 76-9 [Texto en línea]

(36) Johnston JJ. Evaluation of Cocoaand Coffee-Derived Methylxanthines as Toxicants for the Control of Pest Coyotes. J Agric Food Chem 2005; 53: 4069-75 [Texto en línea] 